\title{
LE CONCEPT CRITIQUE DE NATION DE HANNAH ARENDT À LA LUMIĖRE DE SA POLÉMIQUE SECRÈTE AVEC CARL SCHMITT
}

\author{
HANNAH ARENDT'S CRITICAL CONCEPT OF \\ NATION IN LIGHT OF HIS SECRET POLEMIC WITH \\ CARL SCHMITT
}

\author{
Christian Ferrié \\ Université de Strasbourg
}

\section{RÉSUMÉ}

Carl Schmitt ayant interprété de manière nationaliste le concept de nation issu de la Révolution française, Hannah Arendt se voit contrainte de rejeter cette notion dangereuse à cause de sa fatale association à la souveraineté nationale de l'État-Nation et au nationalisme potentiellement raciste qui en ressort. Car le potentiel national-raciste que l'État-Nation a effectivement développé au cours de l'histoire repose sur la fabrication d'un unanimisme national qui permet l'oppression des minorités autant que la destruction de l'espace politique. L'option de la nation républicaine ayant été écartée, le concept de nation ne peut plus être que dans un état critique.

Mots-clefs : nation, État-nation, nationalisme, racisme, révolution nationale

\begin{abstract}
Carl Schmitt having interpreted the concept of nation resulting from the French Revolution in a nationalistic way, Hannah Arendt is forced to reject this dangerous notion because of its fatal association with the national sovereignty of the Nation-State and the potentially racist nationalism which comes from it. For the nationalist potential that the Nation-State has effectively developed over the course of history rests on the fabrication of a national unanimity that allows the oppression of minorities as much as the destruction of the political space. The option of the republican nation having been rejected, the concept of nation can only be in a critical state.
\end{abstract}

Keywords: Nation, State-Nation, Nationalism, Racism, National Revolution 


\section{RESUM}

\section{El concepte crític de nació d'Hannah Arendt a la llum de la seva controvèrsia secreta amb Carl Schmitt}

Havent interpretat Carl Schmitt de manera nacionalista el concepte de nació sorgit de la Revolució francesa, Hannah Arendt es va veure en l'obligació de rebutjar aquesta perillosa noció a causa de la seua associació fatal amb la sobirania nacional de l'Estat-nació i amb el nacionalisme potencialment racista que emergeix. I això perquè el potencial racista nacional que l'Estat-nació ha desenvolupat al llarg de la historia es fonamenta en la fabricació d'una unanimitat nacional que permet tant l'opressió de les minories com la destrucció de l'espai polític. Havent-se rebutjat l'opció de la nació republicana, el concepte de nació no pot estar més que en un estat crític.

Paraules clau: nació, estat-nació, nacionalisme, racisme, revolució nacional 
Hannah Arendt n'envisage pas la nation comme une donnée naturelle et intangible de la vie politique qu'il serait possible de considérer abstraction faite de son histoire. N'est pas tant en jeu le fait que toute nation se constitue au cours du temps, même si Arendt ne peut que rendre justice à cette évidence culturelle. C'est surtout que le concept de nation est historiquement et culturellement déterminé, depuis la Révolution française, par son association au nationalisme et à la souveraineté nationale de l'État-Nation. Dans ces conditions, Arendt ne peut qu'adopter un rapport critique à cette notion bien souvent confondue arec celle de peuple. Ce n'est pas le cas chez Kant qui prend soin de distinguer peuple (populus) et nation (gens) :

«On entend par le mot peuple (populus) la foule d'êtres humains unis sur un territoire dans la mesure où elle forme un Tout. La foule ou bien la partie de la foule qui se reconnaît unie en un Tout civil par son origine commune s'appelle nation (gens), alors que la partie qui se soustrait à ces lois (la foule sauvage au sein de ce peuple) s'appelle populace (vulgus)».'

Si la nation civile et citoyenne $n^{\prime}$ inclut donc pas la canaille du peuple qui ne respecte pas les lois, elle se caractérise néanmoins, en amont de cette civilité assumée, par une provenance ou souche commune (Abstammung) qui renvoie à la racine étymologique de la natio comme issue de la natura: le grec et le latin s'accordent pour enraciner la nation et son génie naturel dans une souche commune (genos vs gens) à l'origine des peuples naturels (ethnè vs nationes) qu'il convient de démarquer du peuple policé des cités

1 KANT, Anthropologie dans une perspective pragmatique (1798), c. "Caractère du peuple »: c'est la définition liminaire. 
grecques tout comme du peuple romain (dèmos vs populus). C'est en ce sens que Hegel entend le terme:

«L'extension de la famille comme passage dans un autre principe est, dans l'existence, pour part l'extension tranquille de la famille en un peuple, une nation, qui a par là même une origine commune naturelle, pour part le rassemblement de communautés familiales dispersées $»{ }^{2}$

Le caractère national contenant le germe qui se développe au cours de I'histoire des nations, il constitue, selon Hegel, une différence aussi durable que la différence raciale : c'est que la constance invariable du climat et des caractères propres à un territoire (désert, proximité ou éloignement de la mer, etc.) contribue à la constance invariable du caractère national. ${ }^{3}$ Mais c'est sans compter sur l'histoire effective qu'Arendt analyse dans les Origines du totalitarisme pour y voir la transmutation nationaliste de la nation souveraine, devenant État-Nation au XIX siècle dans le cadre des sociétés de classe avant de se dissoudre en race transnationale au $x x^{e}$ siècle dans le contexte des sociétés de masse. Ce diagnostic de l'époque à l'origine du concept critique de nation engendre une critique de la violence nationaliste de la Révolution française :

« II importe peu aujourd'hui que le nouvel absolu que l'on a substitué à la domination des monarques absolus ait été, au début de la Révolution, la nation de Sieyès ou bien la Révolution elle-même, quatre ans plus tard sous Robespierre. Car ce qui a mis le monde en flammes depuis lors est la fatale combinaison de la révolution et du nationalisme, la révolution nationale ou le nationalisme révolutionnaire, à savoir un nationalisme qui parle la langue de la Révolution et des révolutions qui déchaînent en premier lieu les passions nationales $»{ }^{4}$

2 HEGEL, Philosophie du droit (1821), remarque du § 181.

3 HEGEL, Encyclopédie des sciences philosophiques (1830), ajout au § 394.

4 Hannah ARENDT (1963: 206, trad. 1967: 232) (" soulevant les masses avec des slogans nationalistes ». Toutes les traductions sont effectuées à partir de la version allemande, bien plus complète : voir Christian FERRIÉ (2008), « Une politique de lecture : Hannah Arendt en allemand », Tumultes, ${ }^{\circ}$ 30, Hannah Arendt abroad, pp. 246-264. 
L'assimilation pour le moins polémique de la Révolution française à une révolution nationale, qui fait immanquablement penser au IIIle Reich et à la France de Vichy, converge avec la compréhension de l'État-Nation comme État national potentiellement nationaliste pour justifier, au nom d'une politique républicaine ouverte à la pluralité des opinions, le rejet arendtien de tout ce qui relève du national. Arendt suppute ainsi dans la volonté générale de Rousseau la matrice antirépublicaine d'un unanimisme national ${ }^{5}$ que Sieyès postule en plaçant la nation au-dessus de toute loi. ${ }^{6}$ Cette lecture au plus haut point problématique est inspirée par l'interprétation tout aussi contestable de Rousseau que Schmitt formule à l'époque de la République de Weimar. ${ }^{7}$

Pour bien comprendre les tenants et les aboutissants de la position prise par Hannah Arendt sur la question nationale, il convient donc de retracer la polémique secrète qu'elle mène contre Carl Schmitt dans l'essai Sur la révolution (1963) après avoir mis soin de préciser au préalable la conception schmittienne de la nation.

\section{LE SENS SCHMITTIEN DE LA NATION}

Selon Schmitt, la Révolution française voit s'affronter deux conceptions antithétiques de la nation: d'un côté, la doctrine démocratique d'inspiration rousseauiste " du peuple (ou plus exactement de la nation) en tant que sujet du pouvoir constituant "; $;$ de l'autre, la doctrine libérale ou antidémocratique de la représentation de la volonté du peuple par l'Assemblée nationale constituante que Sieyès a malencontreusement reliée à la première. ${ }^{9} C^{\prime}$ est en effet à l'occasion de la Révolution que l'idée rousseauiste a pris son essor en corrélation avec la critique de l'absolutisme monarchique par Sieyès : ${ }^{10}$ elle inaugure la forme moderne de la domination démocratique de l'autorité du représentant de l'unité politique du peuple par le pouvoir plébiscitant de la nation qui constitue la substance même de son unité politique dans

5 Ibid., p. 96-99, trad. p. 107-111.

6 Ibid., p. 203, trad. p. 229

7 Voir à ce propos mon article en ligne : "Le Rousseau de Carl Schmitt », Dictionnaire de la réception de J. J. Rousseau, http://rousseaustudies.free.fr/Dictionnairereception.html.

8 Carl SCHMITT, Verfassungslehre (1928), Duncker \& Humblot, 1993, p. 77 (Subjekt der verfassungsgebenden Gewalt), trad. fr. de la Théorie de la constitution, PUF, 1993, p. 213.

9 Ibid., p. 80, trad. p. 216.

10 lbid., p. 77, trad. p. 213. 
l'État. Mais la Révolution est habitée par deux tendances contradictoires qui sont perceptibles chez Sieyès: $:^{11}$ une tendance démocratique qui reste absolutiste, ${ }^{12}$ et une tendance libérale qui aboutit à la monarchie constitutionnelle comme établissement d'une double représentation. Au cours de la Révolution, le peuple s'est ainsi vu dépossédé de son pouvoir par sa double représentation, par le roi et par l'Assemblée nationale, et la démocratie a été abandonnée au profit d'une aristocratie libérale ${ }^{13}$ qui soutient l'État fondé sur la législation au XIXe siècle.

Aux yeux de Schmitt, l'époque libérale a de la sorte entravé l'avènement de la démocratie en tant que forme de l'État, d'une part, en en faisant une méthode de gouverner ou de légiférer ${ }^{14}$ et, $d^{\prime}$ autre part, en confortant le principe de la représentation par l'institution antidémocratique d'une repré-

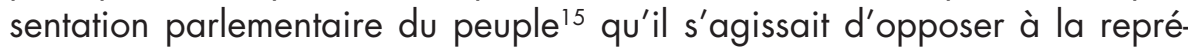
sentation de la nation par le roi. ${ }^{16} \mathrm{C}^{\prime}$ est ce qui explique que la démocratie ne puisse être reconnue à l'époque libérale que comme élément du système parlementaire. ${ }^{17}$ Alors que la forme démocratique de l'État est constituée par l'identité du peuple uni, ${ }^{18}$ la démocratie moderne de masse prend, pendant la période libérale, la forme de la démocratie représentative, un régime mixte qui peut faire figure de compromis avec le passé absolutiste, c'est la monarchie constitutionnelle, ou encore de transition vers l'avenir totaliste : ${ }^{19} c^{\prime}$ est par exemple le régime présidentiel de la République de Weimar qui est scindé entre parlementarisme et démocratie plébiscitaire.

Comme tout État contient en réalité les deux éléments structurels de l'identité immédiate du peuple et de sa représentation, ${ }^{20}$ la forme politique de l'État est en vérité déterminée par la domination d'un principe sur l'autre. Ce qui signifie que la forme politique de l'État peut prendre forme soit à partir du

11 Ibid., p. 80, trad. p. 216.

12 lbid., p. 216 , trad. p. 354.

13 Ibid., p. 80, trad. p. 216.

14 Ibid., p. 224, trad. p. 362.

15 bid., p. 219 , trad. p. 356.

16 Ibid., p. 80, trad. p. 216.

17 Ibid., p. 220 , trad. p. 357.

18 Ibid., p. 223, trad. p. 361.

19 Carl SCHMITT, Der Staat als Mechanismus bei Hobbes und Descartes (1936), Archiv für Rechts- und Sozialphilosophie, t. XXX, 1936/1937, p. 623.

20 Id., Verfassungslehre, p. 205, trad. p. 343 
principe de l'identité substantielle du peuple, soit à partir du principe de la représentation de l'unité politique du peuple par le gouvernement:

«[...] là où le peuple entre en scène en tant que sujet du pouvoir constituant, la forme politique de l'État se détermine à partir de la représentation d'une identité : la nation est là ; elle n'a pas besoin et ne peut être représentée, une pensée qui donne aux exposés souvent cités de Rousseau (Contrat social, III, 15) leur caractère incontestablement démocratique. La monarchie absolve n'est en vérité qu'absolue représentation et repose sur la pensée que l'unité politique est produite seulement par représentation, par présentation. La phrase : "I'État c'est moi" signifie : moi seul représente l'unité politique de la nation $»{ }^{21}$

\section{La distinction politique du peuple comme nation}

L'usage alternatif des termes de peuple et de nation ne signifie pas leur confusion par Schmitt qui conçoit bien plutôt une distinction décisive entre les deux : la nation est la modalité de l'existence proprement politique du peuple. C'est la Révolution qui a révélé le peuple comme nation non plus au sens d'une donnée naturelle, mais au sens du produit historique de la volonté consciente et spirituelle de vivre ensemble :

« La constitution moderne, qui mêle des éléments libéraux et démocratiques, naît pendant la Révolution française de 1789. [...] Le pouvoir constituant présuppose le peuple comme une grandeur politiquement existante ; le mot "nation" désigne de manière significative un peuple éveillé à la conscience politique et capable d'action. [...] le peuple français en tant que porteur du pouvoir constituant se constitue ; il devient conscient de sa capacité d'action politique et se donne à luimême une constitution sur le présupposé, ainsi explicitement affirmé, $d^{\prime}$ 'une unité et d'une capacité d'action politique qui sont bien là. Si le processus fut aussi efficace et énergique, c'est que la décision politique fondatrice consistait à devenir conscient de sa qualité propre en tant que sujet capable d'action et à déterminer lui-même son destin politique. Le peuple français se constitue ainsi de lui-même en un certain sens. En se donnant une constitution, il anticipe déjà l'acte suivant : la décision à prendre sur un type et une forme particulière d'existence. Le peuple devient nation, c'est-à-dire conscient de son existence politique.

21 Ibid., p. 205, trad. p. 342. 
Mais cela ne signifie pas qu'il n'existait pas auparavant et qu'avec la saisie consciente de son pouvoir constituant, il constitue aussi son État. L'être politique précède la donation de constitution $\gg .{ }^{22}$

La substance prépolitique se transforme en sujet politique : le peuple n'est plus simplement constitué, il se constitue comme nation. Cette transfiguration est décisive : issue de la Révolution française, elle semble soumettre la forme de l'État à la volonté du peuple ${ }^{23}$ et imposer la démocratie moderne contre toute domination dynastique. ${ }^{24}$ Mais le processus d'éveil à la conscience politique ${ }^{25}$ est fondé sur un présupposé ${ }^{26}$ dédoublé : I'existence de l'État constitué qui assure l'unité politique du peuple, ${ }^{27}$ dont celui-ci prend conscience comme capacité d'action ; et, par là même, l'existence même du peuple qui ne pourrait pas prendre de décision s'il n'était pas là au préalable. ${ }^{28}$ La décision politique ${ }^{29}$ et totale à propos du type et de la forme $d^{\prime}$ existence politique propre ${ }^{30}$ est prise par le peuple, auto-constitué en nation, ${ }^{31}$ qui est, comme sujet du pouvoir constituant, le fondement originaire de toute la vie politique $:^{32}$

"Selon cette nouvelle doctrine, la nation est le sujet du pouvoir constituant. Nation et peuple sont souvent considérés comme des concepts équivalents, mais le terme "nation" est plus précis et prête moins au malentendu. II désigne en effet le peuple comme unité capable d'ac-

22 lbid., p. 50, trad. p. 183.

23 Ibid., p. 9, trad. p. 137-138 vs. p. 75, trad. p. 209

24 Ibid., p. 81, trad. p. 217.

25 Ibid., p. 50, trad. p. 182; et p. 51, trad. p. 183 (zum politischen Bewußtsein erwacht).

26 Ce qui est présupposé, c'est ce qui existe (bestehend, bestand) au préalable, c'est-à-dire l'existence d'un fond(s) (Bestand: p. 95, trad. p. 232) que la prise de conscience manifeste publiquement (p. 209-210, trad. p. 347).

27 L'État monarchique est le présupposé de la prise de conscience de son identité nationale par le peuple français (p. 50, trad. pp. 182-183 vs p. 79, trad. p. 215).

28 Ibid., pp. 61, 205, 223, trad. pp. 195, 342, 361 (vorhanden).

29 Ibid., p. 76, trad. p. 211

30 Ibid., p. 78, trad. p. 213.

31 lbid., p. 50, trad. p. 183.

32 Ibid., p. 81, trad. p. 217. 
tion politique de par la conscience de sa particularité politique et sa volonté d'exister politiquement, alors que le peuple qui n'existe pas comme nation n'est qu'un rassemblement d'êtres humains liés par des appartenances d'ordre ethnique ou culturelle sans nécessairement exister de manière politique. La doctrine du pouvoir constituant du peuple présuppose la volonté consciente de l'existence politique et, donc, une nation. Ce ne fut historiquement possible qu'après que la France, grâce à la monarchie absolve, soit devenue une unité étatique dont l'existence est présupposée comme allant de soi malgré tous les changements de constitution. Le peuple français $\mathrm{n}^{\prime}$ a trouvé sa forme en tant que nation que dans son existence politique. [...] Le peuple, la nation, reste le fondement originaire, la source de toute force, qui s'exprime dans des formes toujours nouvelles [...] sans pourtant jamais soumettre elle-même son existence politique à une formation définitive $»^{33}$

Au moment de la Révolution, le peuple français se constitue comme nation animée par une volonté d'exister au niveau politique : Sieyès postulant que la volonté de la nation est suffisante, le pouvoir constituant $n$ 'est tenu à aucune procédure juridique. Tout est donc affaire de volonté nationale : «À l'existence politique appartient l'auto-détermination. La constitution au sens positif est une expression de cette possibilité de choisir type et forme de l'existence propre par l'énergie de sa propre décision ». ${ }^{34}$ Or Schmitt comprend l'existence politique dans le sens, $d$ 'inspiration heideggérienne, d'une ek-sistence conçue comme élévation (Heraushebung) à la sphère de l'être public : le politique relevant du « principe spirituel de l'existence politique ${ }^{35}{ }^{35} c^{\prime}$ est-à-dire du principe de la représentation, cet autre type d'être, plus intensif, que permet la représentation politique du peuple est supérieur à d'autres types d'être ${ }^{36}$ qui consistent à être simplement, c'est-à-dire naturellement là ou présent; ce qui revient à dissocier l'existence politique d'un peuple de sa présence naturelle. ${ }^{37}$ Mais Schmitt contredit la conception

33 Ibid., p. 79, trad. p. 215 (Urgrund).

34 bid., p. 71, trad. p. 205.

35 Ibid., p. 212, trad. p. 350 (Vorhandensein).

36 Ibid., p. 210 , trad. p. 347 (andere Arten ihres Daseins).

37 Carl Schmitt démarque sémantiquement l'existence politique de la nation, distinguée par le terme Existenz, de la présence naturelle du peuple, caractérisée comme être-là (da sein) : natürliches Dasein (p. 210, trad. p. 347) vs natürliches Vorhandensein (p. 212, trad. p. 350). 
mécaniste et artificialiste de Hobbes qui semble croire la représentation politique capable de produire l'unité à partir de rien : le néant politique étant l'état de guerre civile dont les Anglais ont fait l'expérience, Hobbes conçoit la naissance de l'État à partir de l'état de nature de manière mécanique sans s'apercevoir que le mécanisme est tout aussi incapable que l'individualisme de la construction contractuelle d'engendrer la totalité de l'État. ${ }^{38}$ $C^{\prime}$ est que, selon Schmitt, l'énergie d'auto-affirmation existentielle du peuple en tant que nation présuppose son identité substantielle comme sub-stance qui soutient au préalable l'autoconstitution de la nation. Schmitt ne cède pas pour autant à une représentation organiciste de la totalité ${ }^{39}$ qui, en raison d'un schéma fonctionnaliste, transformerait les membres du peuple politiquement organisé sous forme d'État en des organes automatisés sans prise sur leur destin. Le peuple devient une nation qui a l'énergie de s'affirmer identique à soi, c'est-à-dire comme unité ayant une identité politique. l'homogénéité nationale du peuple engendre le sentiment de l'unité natio-

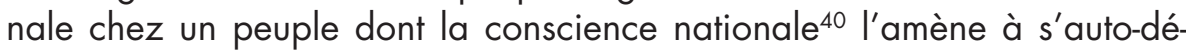
terminer, c'est-à-dire à se donner à soi-même une constitution. Même si la conscience politique de soi et la volonté de sa propre existence politique ne sont pas des données qui seraient d'ordre strictement naturel ou d'origine culturelle ou historique, l'identité du peuple semble bien, quant à elle, avoir pour substance une homogénéité naturellement donnée ou historiquement produite :

«En résumé, l'État repose en tant qu'unité politique sur la relation entre deux principes opposés de configuration, le principe de l'identité (du peuple présent à soi-même en tant qu'unité politique, lorsqu'en vertu de sa propre conscience politique et de sa propre volonté nationale, il a la capacité de distinguer ami et ennemi) et le principe de représentation en vertu duquel l'unité politique est présentée par le gouvernement. La tendance à réduire au minimum gouvernement et direction personnelle réalise le principe $d^{\prime}$ 'identité. Plus ce principe s'impose, plus la résolution des affaires politiques s'accomplit "de soimême" grâce à un maximum d'homogénéité donnée naturellement ou

38 La critique de la conception de L'État comme mécanisme chez Hobbes et Descartes fait l'objet explicite de l'article de 1936 : op. cit., p. 622, 628, 631.

39 Id., Verfassungslehre, p. 56, trad. p. 188, cf. p. 213, trad. p. 351.

40 lbid., p. 65, trad. p. 199. 
advenue historiquement. C'est l'état idéal d'une démocratie tel que Rousseau le présuppose dans le Contrat social. On parle alors de démocratie pure ou immédiate $\gg .{ }^{41}$

\section{La nation entre nature et histoire}

Le spontanéisme qui caractérise à la fois le rapport du peuple à son identité immédiate et la résolution conséquente des affaires politiques s'enracine donc dans l'homogénéité du peuple, qui est fondée sur la substance naturelle ou historique préalable à son existence politique. Ici comme dans les autres passages cités, Schmitt s'appuie sur Rousseau pour étayer sa thèse de l'homogénéité de la volonté générale du peuple souverain : "Le vrai État existe selon Rousseau uniquement là où le peuple est si homogène que règne pour l'essentiel l'unanimité ". ${ }^{42}$ Même s'il semble décisif que le peuple se définisse en son identité substantielle, le fait est que cette auto-détermination revient à une reconnaissance par la conscience (Bewußtsein) de l'être (Sein) préalable qui constitue la substance de l'identité du peuple homogène. Loin d'être formelle et abstraite, l'identité en jeu dans la démocratie est une identité de contenu ayant un fonds substantiel qui fonde une égalité concrète : «le concept démocratique de l'égalité ne se satisfait pas de $n^{\prime}$ importe quelle égalité universelle et indifférente qui est présente de soi sans égard pour la substance et valeur $»{ }^{43}$ Cette identité de substance ou de valeur présuppose la démarcation ${ }^{44}$ ou encore la distinction de l'appartenance à un peuple déterminé :

« [...] la démocratie politique ne peut par conséquent reposer sur l'indistinction de tous les hommes, mais uniquement sur l'appartenance à un peuple déterminé, laquelle appartenance peut être déterminée par des moments très divers (représentations d'une race commune [Rasse], foi, destin commun, tradition) $»{ }^{45}$

41 Ibid., p. 214-215, trad. p. 352.

42 Id., "Der Gegensatz von Parlementarismus und moderner Massendemokratie » (1926), Positionen und Begriffe, Hanseatische Verlagsanstalt Hamburg, 1940, p. 63 (Einstimmigkeit). Trad. fr. de «L'opposition entre parlementarisme et démocratie de masse » dans Parlementarisme et démocratie, Seuil, 1988, p. 111

43 Id., Verfassungslehre, p. 226 (Substanz oder Wert), trad. p. 364.

44 Ibid., p. 226, trad. p. 364.

45 bid., p. 227, trad. p. 365. 
L'appartenance à un peuple donne le sens de son identité immédiate qui est déclinée selon plusieurs registres : identité de race, de foi, d'histoire (passée et à venir) ou de mœurs. Ces moments ne sont que des possibilités dans la mesure où chaque époque a vu la prédominance d'un de ces aspects ${ }^{46}$ constitutifs qui peuvent être déterminants ou être au contraire dominés. Ces moments définissent la substance de l'égalité 47 du peuple : l'être-là (stare) qui soutient et sous-tend toutes les qualités du peuple, les attributs essentiels qui définissent le peuple en sa constitution, fait qu'il est effectivement là (estar) et qu'il se redresse (stasis) face aux autres peuples pour affirmer sa propre valeur. Schmitt distingue cinq sens historiquement donnés à l'égalité substantielle par les diverses démocraties, dont le contenu substantiel est défini à travers la qualification du type d'homogénéité (Gleichartigkeit) qui constitue l'identité du peuple.

a) La démocratie athénienne critiquée par Platon et par Aristote repose sur une homogénéité de type physique et moral, c'est-à-dire fondée sur la différence de nature entre les barbares, les esclaves et les Grecs : I'aretè des Grecs leur donne «le sens d'un type intensifié d'existence ${ }^{48}$ qui les prédispose à l'existence politique..$^{49}$

b) La virtù de Machiavel, la vertu de Montesquieu et surtout la volonté générale de Rousseau permettent de dégager le fondement démocratique de l'État qui repose sur «l'homogénéité ou identité du peuple à soi » : sortant de la même substance, tous veulent la même chose. Cette expression la plus conséquente de la pensée démocratique qui présuppose l'homogénéité complète et substantielle du peuple est à l'origine de la dictature jacobine qui dénie tout civisme à son adversaire hors la loi. ${ }^{50}$

c) La forme moderne de démocratie immédiate qu'ont inaugurée les sectateurs anglais du $\mathrm{XV}^{\mathrm{e}}{ }^{\mathrm{e}}$ siècle, le mouvement des Levellers, repose sur une foi

46 Ibid., p. 228, trad. p. 366 : « la substance de l'égalité peut être différente selon les différentes démocraties et les différentes époques $»$.

47 Ibid., p. 228, trad. p. 366.

48 lbid., p. 228 , trad. p. 366.

49 Ibid., p. 229, trad. p. 366. Citant la République de Platon (VIII, 557c), Schmitt traduit par

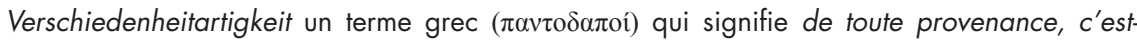
à-dire venant de toutes les terres ( $\delta \dot{\alpha} \pi \varepsilon \delta o v)$ : p. 228, trad. p. 366. La provenance (Herkunff) n'étant autre que le genos, Schmitt va s'efforcer de définir le sens moderne, à l'époque de la démocratie de masse, du genos comme Art (type, espèce) impliquant l'homogénéité : le terme Gleichartigkeit signifie littéralement égalité de type (Gleichheit der Art).

50 lbid., p. 229-230, trad. p. 367. 
commune qui constitue la substance de l'égalité démocratique de la communauté des élus. ${ }^{51}$

d) La démocratie nationale apparue lors de la Révolution française de 1789 présuppose l'homogénéité nationale du peuple: "Nation signifie, par rapport au concept général de peuple, un peuple individualisé par sa conscience politique particulière ».52 Reprenant les analyses de Renan sans le citer, Schmitt explique que l'unité de la nation et la conscience de l'unité de la nation peuvent comprendre différents éléments parmi lesquels sont déterminants, plus que la communauté linguistique, la communauté de destin passé et la volonté d'une communauté de destin à venir. ${ }^{53}$ La conséquence pratique est le principe de nationalité comme présupposé d'un État nationalement homogène. Dans le cas d'un État composé de plusieurs nations, le droit des gens qui entend garantir la protection des minorités préconise de produire l'homogénéité nationale par le moyen pacifique d'une assimilation à la nation dominante. Mais il existe une méthode violente, bien plus rapide : "oppression, déportation de la population hétérogène et autres moyens radicaux ${ }^{54}$ comme l'extermination. ${ }^{55}$ Le contrôle de l'immigration, la domination coloniale de populations hétérogènes sans annexion du territoire colonisé, la protection de l'industrie nationale contre le capital étranger, la nationalisation des ressources naturelles du pays et la déchéance de nationalité sont tout autant de moyens d'assurer l'homogénéité nationale.

e) La politique bolchevique substitue à l'homogénéité nationale l'homogénéité d'une classe sociale, le prolétariat, sur le fondement de la solidarité entre les travailleurs de toutes les nations contre les bourgeois: I'opposition entre États prolétariens et capitalistes qui se substitue aux oppositions nationales fait que la démarcation entre amis et ennemis gagne en intensité $; 56$ cessant d'être d'ordre économique, la classe est transformée par la lutte politique en « peuple de l'État ». ${ }^{57}$ Comme le concept politique de démo-

51 Ibid., p. 230, trad. p. 368.

52 Ibid., p. 231 , trad. p. 369.

53 Ibid., p. 231, trad. p. 369. Voir Ernest RENAN (1882), Qu'est-ce qu'une nation ?, 1992, p. 54.

54 lbid., p. 232, trad. p. 369.

55 Id., "Der Gegensatz von Parlementarismus und moderner Massendemokratie », op. cit., p. 59, trad. p. 106. La traduction française de Vernichtung par exclusion est un euphémisme.

56 Id., Verfassungslehre, p. 234, trad. p. 371

57 Ibid., p. 233-234, trad. p. 371. 
cratie et le concept démocratique de peuple ne sont pas affectés par cette transformation, cette critique larvée du léninisme signifie que, pour Schmitt, l'opposition nationale entre peuples homogènes est indépassable. ${ }^{58}$

Dans ces conditions, l'idée même de démocratie nationale implique et exige une politique d'homogénéisation qui consiste à ramener la nation à son identité nationale : le glissement de la nation depuis le sens politique d'un peuple conscient de soi jusqu'au sens prépolitique de l'homogénéité nationale d'une souche (genos) est au cœur de la récupération schmittienne du concept révolutionnaire de nation républicaine. Si l'événement historique par lequel un peuple devient une nation consciente d'elle-même repose bien sur une décision existentielle d'ordre politique, le type d'existence politique qui en ressort reste toujours, pour Schmitt, enraciné au préalable dans l'effectivité concrète d'un peuple particulier, comme la nation suisse ou française :59 l'existence politique est fondée dans le type propre ${ }^{60}$ du peuple, par exemple allemand ${ }^{61}$ ou français. ${ }^{62}$ En 1923, Schmitt évoquait déjà les différents éléments actifs dans le sentiment national des divers peuples :

«Les représentations les plus naturelles [naturhaff] de la race [Rasse] et de l'origine [Abstammung], un "terrisme" apparemment plus typique pour les tribus [Stämme] celto-romaines; ensuite, langue, tradition, conscience culture et civilisation, conscience d'une communauté de destin, une sensibilité pour l'être-différent en soi - tout ceci se meut aujourd'hui plutôt dans la direction d'oppositions nationales que de classes $\gg .{ }^{63}$

58 Id., Theorie des Partisanen (1963), Duncker \& Humblot, 1995, pp. 61-63 vs pp. 91-94, trad. fr. Théorie du partisan, CALMANN-LÉVY (1972), pp. 270-272 vs pp. 305-309. Schmitt y reprochera à Lénine d'avoir absolutisé l'ennemi en déniant la dimension effective de l'opposition tellurique entre des territoires nationaux, là où Mao s'est contenté de reconnaître l'ennemi effectif, tellurique : voir Christian FERRIÉ (2017), "Visions nationalistes du bolchevisme », La pensée, $n^{\circ} 390$, pp. 103-105.

59 Id., Verfassungslehre, p. 23, trad. p. 153, cf. p. 15, trad. p. 145.

60 Ibid., p. 207, 82, trad. p. 344, 218 (Eigenart). II en ressort que le type propre de Constitution ressort de la constitution déterminée du peuple : par exemple, Schmitt parle du « type propre de cette constitution monarchique de style allemand » (p. 55, trad. p. 187).

61 Ibid., p. 95, trad. p. 233.

62 Ibid., p. 50, trad. p. 183 (neve Art politischen Daseins).

63 II s'agit d'une recension des Réflexions sur la violence (1908) de Georges SOREL : "Die politische Theorie des Mythus » (1923), Positionen und Begriffe, op. cit., p. 16-17. Les repré- 
Loin que la volonté spirituelle de la nation permette de dissoudre les différences préalables (race, langue, religion, économie, territoire), ${ }^{64}$ la participation démocratique des citoyens de l'État a pour sens de permettre « la production de l'identité » du peuple en son unité politique. ${ }^{65}$ Mais cet engagement politique de la nation ne crée pas ex nihilo l'identité nationale qui est toujours déjà là : 66 la politique d'homogénéisation reproduit bien plutôt l'homogénéité nationale en se démarquant des nations étrangères ou en discriminant les éléments hétérogènes qui la mettent en péril en menaçant sa pureté. $C^{\prime}$ est que la conscience politique ${ }^{67}$ de l'identité nationale détermine la volonté de se différencier ${ }^{68}$ et, donc, de discriminer l'élément étranger qui, de l'intérieur ou de l'extérieur, menace l'identité propre de la nation. ${ }^{69}$

Les diverses modalités de la conscience d'appartenir à un même peuple impliquent toutes l'homogénéité comme constitutive du peuple : I'identité du peuple, c'est son homogénéité qui le distingue en tant que peuple déterminé au sein du genre humain. Pour Schmitt, le « monde politique est un pluri-versum » constitué par « différents peuples, religions, classes et autres groupes humains $» .^{70} \mathrm{Ce}$ partage du monde est tout aussi bien racial que linguistique ou juridique : "le monde de l'esprit objectif est un monde pluraliste : pluralisme des races [Rassen] et peuples, religions et cultures, langues et systèmes de droit ». ${ }^{71}$ L'homogénéité est ainsi constituée par l'appartenance à un même genos au sens de la race, nation, tribu ou encore de l'espèce. L'appartenance à un peuple se manifeste ainsi par le sentiment d'être de la

sentations d'une race commune sont des représentations mythologiques dans la mesure même où, par différence avec la foi, le destin commun et la tradition qui sont actuellement présents c'est-à-dire vécus, l'origine de l'identité ne peut être que représentée.

64 RENAN, Qu'est-ce qu'une nation?, pp. 45-55.

65 SCHMITT, Verfassungslehre, p. 216 (Herstellung der Identität), trad. p. 353

66 Christian FERRIÉ (2007), "Le nationalracisme de Carl Schmitt», Cahiers philosophiques, $n^{\circ} 109$, La voix, pp. 62-67.

67 SCHMITT, Verfassungslehre, p. 231 (Sonderbewußtsein), trad. p. 369.

68 Ibid., p. 229, trad. p. 367 (differenzierend).

69 Id., Der Begriff des Politischen (1932), Duncker \& Humblot, 1963, p. 27, trad. fr. La notion de politique, Calmann-Lévy, 1972, p. 67.

70 lbid., p. 54, trad. p. 97.

71 Id., p. 141, Staatsethik und pluralistischer Staat (1930), Positionen und Begriffe, trad. de «Éthique de l'État et État pluraliste » dans Parlementarisme et démocratie, p. 144. L'absence d'article devant peuples et cultures indique l'intrication, d'une part, entre races et peuples et, $d^{\prime}$ autre part, entre religions et cultures. 
même race (genos) ou encore de la même espèce (Art). L'identité se joue à l'origine sur le sol de l'appartenance commune à une même race ou espèce : I'identité du peuple est homogénéité, c'est-à-dire identité du genos comme espèce (Gleichartigkeit) ou encore identité d'espèce (Artgleichheit) ou de race. Dans État, Mouvement, Peuple (1933), Schmitt conçoit désormais I'homogénéité spécifique du peuple allemand comme identité de la race (Rasse) :

«Si, lors du Congrès national-socialiste des juristes allemands de Leipzig en 1933 dans le grandiose discours final du Führer [...], la pensée de la race a été constamment placée au centre, ce n'était pas un postulat théoriquement inventé. Sans le principe de l'identité de race, l'État national-socialiste ne pourrait subsister et la vie de son droit serait impensable ${ }^{72}$

Conformément à la sémantique omniprésente de la stabilité, l'État (Staat) ne peut se maintenir (bestehen) comme unité politique du peuple qu'en se tenant sur ce fondement statique ${ }^{73}$ qu'est la sub-stance du peuple $^{74}$ allemand dont l'identité se distribue à même la diversité des régions, des peuples (Stämme vs Stamm/ ${ }^{75}$ et des états (Stände), comme l'ordre des fonctionnaires et des juristes $^{76}$ qu'il $^{\prime}$ 'agit de restaurer en les purifiant des éléments étrangers (fremdgeartef)..$^{77}$ Le principe de l'identité de race sans lequel l'État total du Führer ne pourrait subsister un seul jour ${ }^{78}$ est un concept fondamental du droit national-socialiste dans la mesure où la discrimination entre étrangers (Artfremde) et compatriotes ou congénères (Volksgenosse) est fondatrice de la distribution inégale des droits entre les égaux entre eux (Gleichen) et les autres $^{79}$ qui ne leur sont pas égaux (Nicht-Gleiche).

72 Id., Staat, Bewegung, Volk, Hanseatische Verlagsanstalt Hamburg, 1933, p. 42 (Artgleichheit), trad. fr. État, Mouvement, Peuple, Kimé, 1997, p. 59.

73 Id., Legalität und Legitimität (1932), Duncker \& Humblot, 1998, p. 29.

74 Id., Staat, Bewegung, Volk, p. 42, trad. p. 59.

75 Id., p. 19, trad. p. 32.

76 Ibid., p. 44, trad. p. 63.

77 Ibid., p. 19, trad. p. 32.

78 Ibid., p. 46, trad. p. 63.

79 Ibid., p. 19, trad. p. 32. 
La traduction nationalraciste des concepts de la démocratie nationale en confirme la signification polémique. Pour Schmitt qui joue du double sens de gleich (égal vs identique), toute démocratie présuppose la discrimination du dèmos comme communauté des identiques ou égaux (entre eux) par opposition aux autres : égaux par leur identité substantielle à l'intérieur du cercle des identiques, ${ }^{80}$ c'est-à-dire par leur appartenance au peuple (compris notamment comme race), ces identiques sont égaux entre eux en tant que citoyens, ${ }^{81}$ c'est-à-dire par leur appartenance à l'État comme unité politique du peuple. L'identité (Gleichheit) comme homogénéité de substance est la substance préalable qui fonde l'égalité (Gleichheit) de droit, l'égalité démocratique c'est-à-dire l'identité de citoyen étant la condition préalable de toutes les autres égalités légalité devant la loi, droit égal de voter, conscription, accès égal aux postes administratifs). ${ }^{82}$

Le concept démocratique de la nation républicaine est en vérité absorbé par la logique identitaire de l'identité nationale du peuple. La nation politiquement consciente de soi que la Révolution avait révélée voit sa capacité d'action politique réduite à l'approbation plébiscitaire de l'autorité, c'est-à-dire à l'incapacité politique d'un peuple destiné à se soumettre au pouvoir. Loin d'être effectivement sujet du pouvoir constituant, le peuple de la démocratie totale ${ }^{83}$ est bien plutôt sujet au pouvoir constitué de l'autorité représentative de l'unité politique du peuple. Défini positivement par son identité substantielle, ce peuple est défini par la négative en tant que «masse inorganisée » des non privilégiés, non distingués, non possédants qui ne gouvernent pas plus qu'ils n'administrent : la présence effective sur la place publique d'un peuple (Rousseau) qui n'existe que dans l'espace public se réduit néanmoins à acclamer un meneur ou une proposition ${ }^{84} \mathrm{Le}$ plébiscite comme décision du peuple (Volksentscheid) est la seule et unique activité de cette masse inorganisée :

« Le sens de l'expression plébiscitaire de la volonté n'est pas de normer, mais de décider par la volonté. Il est dans la nature des choses que des plébiscites ne puissent être mis en place que de manière inter-

80 lbid., p. 60, trad. p. 108.

81 Verfassungslehre $\{1928\}$, p. 227, trad. p. 365 (Théorie de la constitution).

82 lbid., p. 227, trad. p. 365.

83 Christian FERRIÉ (2010), "Carl Schmitt, la représentation national(rac)iste du peuple », in Yves VARGAS (dir.), De la puissance du peuple, t. Iv, Le temps des Cerises, pp. 230-235.

84 SCHMITT, Verfassungslehre, pp. 241-243, trad. pp. 380-382. 
mittente ; on ne peut pratiquement pas organiser le fameux "plébiscite de tous les jours". Le peuple ne peut dire que oui ou non; il ne peut conseiller, délibérer ou discuter ; il ne peut pas gouverner, ni administrer ; il ne peut pas non plus produire de norme, mais uniquement sanctionner par un oui une proposition de norme qui lui est présentée. Surtout, il ne peut pas même poser de question, mais uniquement répondre par oui ou non à une question qui lui est proposée $»{ }^{85}$

C'est la quintessence du troisième sens du peuple comme " grandeur non formée »: la masse informe et uniforme n'est capable de décider que sous la forme $d^{\prime}$ un plébiscite ( $c^{\prime}$ est le sens $1 \mathrm{c}$ ). ${ }^{86}$ Le peuple en tant que nation (c'est le sens la du peuple comme sujet du pouvoir constituant) ne fait donc qu'acclamer la Constitution proposée par l'élite démocratique issue du peuple. En tant que porteur de l'opinion publique, le sujet des acclamations qu'est le peuple ( $c^{\prime}$ est le sens $1 \mathrm{~b}$ ) s'avère être une grandeur négativement déterminée : Schmitt ayant rejeté la fiction libérale d'un peuple constitutionnellement formé, il ne reste qu'une foule ou masse de personnes, qui, quelles que puissent être les prédispositions substantielles du peuple (son identité comme homogénéité naturelle ou historique), ne peut finalement accéder à l'existence politique que par la représentation politique.$^{87}$ La nation souveraine dont Schmitt emprunte la notion à Sieyès et Rousseau n'est en fin de compte qu'une fiction qui dissimule une plèbe acclamant le pouvoir en place.

\section{LA CRITIQUE ARENDTIENNE DU CONCEPT DE NATION}

Hannah Arendt développe, de son propre point de vue, un concept critique de nation qui prend le contrepied de la position adoptée par Carl Schmitt. Dès les années 1930, Arendt aborde la question nationale sous I'angle de l'antisémitisme, c'est-à-dire du rapport polémique d'une nation dominante à une minorité nationale, en prenant tout $d$ 'abord dans Rahel Varnhagen la perspective d'une juive allemande de l'époque romantique.

Selon Herder, la fixation des Juifs sur leur histoire et leur religion passées en fait un peuple asiatique qui est resté étranger en Europe : signe d'un at-

85 Id., Legalität und Legitimität, p. 86.

86 ld., Verfassungslehre, p. 251, trad. p. 390

87 Ibid., p. 212, trad. pp. 349-350. 
tachement national, " la religion étrangère devient la religion d'une nation autre $»{ }^{88}$ De ce fait, il convient à ses yeux de déplacer la question juive du terrain de la tolérance religieuse sur le plan de l'émancipation civile de la nation juive qui a dû jusqu'à présent s'adapter de manière parasitaire à un monde étranger : il s'agit donc de radicaliser l'exigence d'assimilation en proposant $d$ 'intégrer cette nation autre, étrangère, à l'Allemagne, ${ }^{89} c^{\prime}$ est-àdire " $d^{\prime}$ incorporer une autre nation au peuple allemand et à l'Europe ${ }^{90}$ Mais la dissolution du statut privilégié des Juifs (d'exception) au sein de «la nation toute entière » revient à " extirper le Juif en nous » du point de vue de Rahel qui s'assimile à travers les Discours à la nation allemande de son ami Fichte, proche de "l'antisémitisme patriotique » qui sévit à cette époque. ${ }^{91}$ Selon Arendt qui parle désormais de peuple juif à la fin des années 1930,92 il existe une solution politique permettant d'éviter une assimilation qui équivaut à renoncer au judaïsme.

\section{La fédération des nations}

En janvier 1940, Arendt envisage l'émancipation nationale du peuple juif dans le cadre d'un système fédéral en Europe où l'appartenance à un territoire serait remplacée par l'appartenance à une union de nations où seule l'union en tant qu'ensemble serait un acteur politique : " une politique européenne donc avec maintien simultané de toutes les nationalités » pour lesquelles un droit des minorités retrouverait son sens. ${ }^{93} \mathrm{C}^{\prime}$ est que la « collusion entre l'État et la Nation » au XIXe siècle, qui a obligé les Juifs à

88 Hannah ARENDT, «Aufklärung und Judenfrage » (1932), Die verborgene Tradition, Suhrkamp, 1976, p. 120.

$89 \mathrm{Ibidem}$.

90 Id., Rahel Varnhagen, Piper, 2003, p. 45, trad. de l'allemand, "Petite Bibliothèque Payot », 2016, p. 56 (einverleiben est traduit par intégrer).

91 Ibid., p. 139 vs pp. 142-143 (die ganze Nation), trad. p. 177 vs pp. 181-182.

92 Ibid., pp. 226-227, trad. pp. 289-290. Pour la datation des deux derniers chapitres, voir I'avant-propos (p. 9, trad. p. 11). Dans les deux derniers chapitres de Rahel Varnhagen, écrits à Paris à la fin des années 1930, l'expression de peuple juif (confronté à ses ennemis) fait son apparition : pp. 226-227, 230-231, trad. pp. 289-290, 294-295. L'idée d'une « masse obscure du peuple » (die dunklen Masse des Volkes) décrit une réalité sociale, la masse des Juifs pauvres (p. 227, trad. p. 290) plutôt qu'elle ne désigne une entité politique : celle d'un peuple international confronté à ses ennemis (pp. 230-231, trad. pp. 294-295).

93 Voir la lettre d'Arendt à Erich Cohn-Bendit de janvier 1940 : Vor Antisemitismus ist man nur noch auf dem Monde sicher, Piper, 2000, p. 231, trad. de «À propos du problème des minorités », in Étienne TASSIN et allii (2007), Hannah Arendt. Crises de l'État-Nation, Sens\&Tonka, 2007 , p. 374. 
s'assimiler, a engendré un nationalisme dont les horribles transferts de population et les massacres divers, des Arméniens aux pogroms ukrainiens, ont été l'ultime conséquence au $x x^{e}$ siècle. ${ }^{94}$ En revanche, le Commonwealth anglais indique un autre modèle dans lequel on ne cesse pas d'être Indien ou Canadien lorsqu'on appartient à l'Empire britannique..$^{95}$ II ne s'agit donc pas de croire à l'utopie d'une nation européenne unitaire, mais d'espérer " une reconnaissance du peuple juif » qui passerait par sa représentation au sein d'un parlement européen » :

«Cela ne me semble pas être une utopie que d'espérer en la possibilité $d^{\prime}$ une fédération de nations avec un parlement européen. [...]. Dans une telle fédération, nous pourrions être reconnus en tant que nation européenne avec représentation dans un parlement européen. Pour cette "solution" de la question juive, le jeu du miroir déformant $d^{\prime}$ un peuple sans terre qui cherche une terre sans peuple - c'est-à-dire la lune ou encore la délivrance de la politique par le folklore - perdrait enfin tout sens $» .96$

Comme il n'y a rien à attendre d'une politique des minorités, les Juifs doivent rechercher la solidarité du côté non des minorités mais des petites nations européennes dont l'existence territoriale est menacée : en tant que membre de l'union des nations européennes et d'un État européen, la nation juive pourrait peut-être chercher une région de colonisation ou garder effectivement la Palestine à condition qu'une telle colonie à l'extérieur de l'union soit garantie par cette union. ${ }^{97}$ Bien que la corrélation entre État-Nation et nationalisme soit déjà évoquée, le concept de nation n'est pas encore devenu critique dans la mesure où Arendt s'inscrit, dans les années 1940, dans la logique d'un mouvement de libération nationale du peuple juif qu'attestent les articles publiés dans le journal new-yorkais Aufbau.98

94 lbid., p. 231, trad. p. 374.

95 Ibid., p. 231, trad. p. 375.

96 Ibid., p. 232, trad. p. 375.

97 Ibid., p. 234, trad. p. 377.

98 Engagée comme éditorialiste en novembre 1941 par le journal de langue allemande Aufbau, Arendt écrit un article en allemand tous les quinze jours de 1941 à 1942, puis de manière moins régulière jusqu'à 1945 : Marie Luise Knott a réédité ces articles dans Vor Antisemitismus ist man nur noch auf dem Monde sicher, Piper, 2000, p. 73 (17-07-42) vs pp. 98-99 (9-1 1-42). Il en existe une traduction partielle en français dans Auschwitz et Jérusalem, Presses Pocket, 1993. 
Si la «souveraineté de l'État-Nation » qui décidait souverainement d'attribuer ou non la citoyenneté de l'État a démontré sa propre absurdité en livrant des centaines de milliers de ses citoyens à " l'arbitraire souverain d'autres nations", le sort des apatrides " exclus de la vie nationale des peuples » au $\mathrm{Xx}^{\mathrm{e}}$ siècle fait écho au destin du peuple juif au $\mathrm{XIX}^{\mathrm{e}}$ siècle, le premier peuple européen à être déclaré paria : maintenant que toutes les nations européennes sont devenus des peuples parias sans droits, c'est l'occasion pour le peuple juif d'entrer dans le concert des nations en luttant pour son émancipation au sein même du combat pour la libération de l'Europe. ${ }^{99}$ Dans le contexte où l'antisémitisme nazi menace effectivement l'existence du peuple juif, Arendt considère que " la libération nationale du peuple juif » passe par la participation active d'une armée juive à la guerre. ${ }^{100}$ Son plaidoyer en faveur du "mouvement national juif », qu'il faudrait conforter contre les ploutocrates par un véritable mouvement révolutionnaire du peuple, est tout sauf nationaliste : c'est qu'à ses yeux l'antisémitisme ne peut être liquidé, comme aux États-Unis, qu'en corrélation avec une «solution juste et très moderne de la question des nationalités $»{ }^{101}$

Cela passe par un changement de paradigme conceptuel qui rende justice à la catastrophe française : I'occupation allemande de Paris prouve très clairement le " déclin de la nation dans son ancienne forme ». ${ }^{102}$ Cette recension de Simon (1942) fait écho au diagnostic antérieur d'Arendt : Hitler a commencé par "liquider la nation allemande » en lui substituant le sang, la race et l'empire, avant de menacer de déclin « la nation française qui, née de la Révolution, était devenue la nation européenne par excellence ». ${ }^{103}$ Dans le contexte du déclin de l'État-Nation et de l'État de droit que prouve la persécution actuelle des Juifs, l'indignation du peuple français contre les déportations de Juifs est néanmoins le premier signe d'un réveil de la conscience nationale de peuples sortant des ruines du déclin national un peu partout en Europe, de sorte qu'une même «volonté politique » de redevenir une nation pourrait unir tous les peuples:

99 Ibid., pp. 25-28 (28-11-1941).

100 lbid., p. 73 (17-07-1942).

101 Ibid., pp. 99-100 (06-11-1942).

102 lbid., p. 113 (26-03-43).

103 lbid., pp. 90-91 (25-09-1942). 
«La catastrophe européenne a provoqué le déclin non seulement des anciens États-nations, mais encore des conflits et des différences entre les peuples qui étaient parvenus à devenir des nations et les autres qui étaient restés "seulement" des peuples; tous sont redevenus ensemble de "simples" peuples ». ${ }^{104}$

Associant nation et État, Arendt semble ainsi assimiler l' "émancipation nationale » à la création d'un État. Mais l'échec patent de la Société des Nations à résoudre le problème national sur la base d'États souverains et de formations multinationales protégeant les minorités invite à trouver une autre solution, fédérale : pour la Palestine, Arendt plaide à plusieurs reprises pour une intégration à un Commonwealth britannique rassemblant les nations anglo-saxonnes et tous les peuples jusqu'alors dominés dans le cadre de l'empire colonial ${ }^{105}$ en un " véritable Commonwealth des $\mathrm{Na}$ tions ». ${ }^{106}$ Si beaucoup des Juifs qui combattent les armes à la main en Pologne partiront volontairement en Palestine pour obtenir « liberté et égalité de droit en tant que nation et sécurité en tant que peuple ", tous les Juifs qui resteront en Europe devront jouir d'un « même statut de la nationalité juive » qui garantira " l'intégration du peuple juif à la communauté future des peuples européens ». ${ }^{107}$ Cette solution d'une fédération inter-nationale au sein de l'Europe, qui découplerait la nationalité du territoire pour en faire un statut personnel, est mentionnée dans une recension de $1946^{108}$ qui préfigure l'argument qu'Arendt développera dans les Origines du totalitarisme : le nationalisme résulterait de la conquête de l'État par la nation qui est à la source de l'État-Nation du XIXe siècle. ${ }^{109}$

104 Ibid., p. 93.

105 Ibid., pp. 122-123 (17-12-1943), trad. pp. 62-63. (17 \& 31-12-1943). Dans la seconde partie de la contribution, publiée en anglais dans Aufbau le 31 décembre 1943, Arendt envisage également l'intégration de la Palestine à une Fédération méditerranéenne : celle-ci pourrait être élargie à une plus grande Fédération des nations européennes qui reconnaîtrait la Palestine comme foyer national pour les Juifs européens et du monde entier (pp. 123-125, non traduit en français dans Auschwitz et Jérusalem).

106 Ibid., p. 141, cf. p. 143 (02-06-44).

107 Ibid., p. 132 (21-04-44).

108 Id., recension de The Nation (janvier 1946), Essays in Understanding, Schocken Books, 1994 , p. 210.

109 Ibid., p. 208. Dans The Origins of Totalitarianism (1951 vs 1958), Arendt cite Delos : la conquête de l'État par la nation (p. 489, trad. p. 511 ) l'empêche de garder sa position d'État au-dessus des classes et des partis (p. 750, trad. p. 674). Les citations sont des traductions de 


\section{Nationalisme de l'État-Nation}

Contre la confusion habituelle entre peuple et nation, entre nationalisme et patriotisme, l'ouvrage de J. T. Delos intitulé La Nation (Montréal, 1944) a l'insigne mérite, selon Arendt, de définir les termes qui permettent de cerner le problème de notre temps : la réalité politique étant basée sur des nations menacées par le nationalisme, comment conjurer ce développement fatal ? $^{110}$ Comprendre que le problème naît de l'État-Nation présuppose de distinguer au préalable nation et État : un peuple devient une nation en prenant conscience de soi à travers son histoire, laquelle histoire a laissé des traces sur le territoire auquel la nation est attachée en tant que société fermée hostile aux migrations où règne le droit du sang ; étranger au corps national, l'État est une puissance qui tend à la conquête agressive de territoires. ${ }^{111}$ Pourtant, ce qui constitue "le mal de notre temps», ce n'est pas la déification de l'État, mais la nation ${ }^{112}$ qui s'empare de l'État comme instrument pour défendre la souveraineté nationale : le nationalisme provient en effet de «l'identification de l'État et de la Nation » en un État-Nation qui garantit les droits des seuls nationaux et s'octroie le droit national à l'expansion impérialiste avant d'aboutir aux « formes totalitaires de nationalisme ». ${ }^{13} C^{\prime}$ est que le sentiment national ne sert plus simplement de ciment à une société libérale menacée par une atomisation individualiste, comme ce fut le cas au XIXe siècle: au Xxe siècle, l'État-Nation prend désormais la figure d'un État totalitaire qui rend le nationalisme fasciste. ${ }^{114}$

Dans les Origines du totalitarisme (1951), Arendt reprendra le diagnostic de cette maladie du XIX siècle $^{115}$ qui empire pendant l'époque impérialiste ${ }^{116}$ avant que le déclin ne s'achève en effondrement lors de la seconde

I'allemand: Elemente und Ursprünge totaler Herrschaft (1955) Piper, 1986, trad. fr. à partir de l'anglais dans Les origines du totalitarisme. Eichmann à Jérusalem, Gallimard, "Quarto », 2002.

110 Ibid., p. 207.

111 lbid., p. 208.

112 lbid., p. 209.

113 lbid., pp. 208-209.

114 Ibid., p. 209.

115 ld., Les origines du totalitarisme, p. 221, trad. p. 433. Le indique que le passage invoqué n'existe pas dans la version américaine et, donc, dans la traduction française.

116 Ibid., p. 744, trad. p. 669 («L'impérialisme prépare le déclin de l'État-Nation »). 
guerre mondiale. Pour Arendt, l'essor et le déclin de l'État-Nation a constitué la splendeur et la misère de la modernité politique: ${ }^{117}$ la splendeur, $c^{\prime}$ est le principe de l'égalité de droit de tous les citoyens; ${ }^{118}$ la misère, c'est l'exclusion hors de la communauté homogène de la nation des éléments hétérogènes (comme les Juifs) ${ }^{119}$ qui finit par se manifester par un chauvinisme sauvage ${ }^{120}$ qui va sombrer dans le racisme national ou supranational. Les Juifs ayant toujours refusé de "se laisser nationaliser et assimiler », les nationalistes les ont accusés d'être un " corps étranger dans la famille des peuples européens ${ }^{121}$ alors qu'ils ne formaient en réalité qu'un «corps fermé à l'intérieur de la nation $» .^{122}$

Avant de dégénérer en racisme, ${ }^{123}$ le nationalisme européen s'est laissé contaminé par l'infection impérialiste : la lutte des classes ayant fissuré le sentiment national et fait éclater "le corps de la nation », I'expansion parut réunifier « la nation éparse » de sorte que les impérialistes, qui parasitaient le patriotisme en invoquant la pérennité de la nation, devinrent aux yeux des patriotes un « dangereux ennemi à l'intérieur de la nation ». ${ }^{124}$ Car la domination bureaucratique de l'Inde provoque une discrimination raciale qui menaçait en retour de " transformer toute la nation en faisant d'un peuple une race $» .^{125}$ Néanmoins, la relative modération de la domination anglaise qui a constamment respecté un minimum de droits de l'homme a non seulement empêché la " destruction de l'État-Nation en métropole », mais encore préparé « la seule nouvelle fondation politique de ce siècle : la transformation de la nation anglaise [...] en un Commonwealth des peuples anglais ». ${ }^{126}$ L'Angleterre est le seul pays qui a pu liquider ses acquisitions impérialistes sans que l'effet boomerang ne se solde par la destruction de « la structure politique de la nation », alors que l'Europe continentale a subi

117 Ibid., pp. 212-213, trad. p. 327.

118 Ibid., p. 88, trad. p. 253.

119 Ibid., p. 114, trad. p. Z68, cf. p. 126, trad. p. 276 (il est question des Juifs alsaciens comme élément hétérogène dans la France homogène).

120 Ibid., p. 69, trad. p. 242.

121 Ibid., p. 71, trad. p. 243.

122 Ibid., p. 138, trad. p. 283.

123 Ibid., p. 356, trad. p. 419.

124 Ibid., p. 342, trad. p. 407 , cf. p. 346, trad. p. 410.

125 Ibid., p. 403, trad. p. 448.

126 Ibid., p. 471, trad. p. 499. 
de plein fouet l'influence de groupes annexionnistes, comme la mouvance pangermaniste, qui tentaient $d^{\prime}$ '« impérialiser la nation » en la mettant au service de l'oppression d'autres peuples: ${ }^{127}$ en Allemagne, ce nationalisme raciste (völkisch) qui s'est substitué à l'émancipation nationale ${ }^{128}$ a mobilisé la « conscience nationale raciste des Allemands » en vue des guerres nationales. ${ }^{129}$ En France, Arendt décèle au XVIII siècle dans I'opposition de la race aristocratique à la nation citoyenne les premiers germes du « concept de race qui a détruit la nation et anéanti l'idée d'humanité née de la nation $» .{ }^{130}$

Dans le chapitre consacré au "Déclin de l'État-Nation », Arendt enracine la déception des peuples auxquels la souveraineté nationale et l'autodétermination n'ont pas été accordées dans l'acceptation de la matrice théorique qui prend l'autodétermination nationale pour un droit de l'homme: " c'est la Révolution française qui a fondé la tradition des États-Nations en identifiant la souveraineté nationale et la jouissance des droits de l'homme ».131 Le conflit latent entre État et Nation provient de cette confusion entre la déclaration universelle des droits de l'homme et la revendication particulière de «droits spécifiquement nationaux d'un peuple souverain qui les devait à sa lutte de libération et à son histoire nationale », dans la mesure où la souveraineté de l'État chargé de faire respecter les droits nationaux a de plus en plus perdu le caractère d'un État de droit rationnel fondé sur la volonté populaire pour être divinisé de manière romantique comme incarnation pseudo-mystique de l'âme nationale :

« Là où le nationalisme dépassa les limites tracées par le patriotisme, il fut l'expression de ce processus par lequel l'État a été perverti en instrument de la nation et le citoyen de l'État confondu avec le membre d'une nation déterminée ${ }^{132}$

127 Ibid., p. 347, trad. p. 411.

128 lbid., p. 365, trad. p. 424.

129 lbid., p. 368, trad. p. 427.

130 Ibid., p. 358, trad. pp. 419-420.

131 Ibid., p. 570, trad. pp. 567-568.

132 Ibid., p. 490, trad. p. 512. Dans la réécriture allemande de 1955 du texte de 1951 apparaît plusieurs fois la notion de volonté du peuple (souverain). 
Alors même qu'elle est née de la souveraineté d'un peuple libre, la souveraineté de l'État-Nation a cessé d'être l'expression de la liberté du peuple. La conception nationaliste de la souveraineté nationale constitue bien une perversion du patriotisme républicain. Ce n'est pas tant la souveraineté populaire en tant que principe républicain que critique Arendt, c'est bien plutôt la souveraineté nationale en tant que principe nationaliste de l'État-Nation. Le concept de nation aurait donc deux sens opposés : le peuple au sens politique et moderne d'une nation républicaine ouverte à l'assimilation des non-nationaux s'opposerait en tout point au « corps essentiellement homogène de la nation ${ }^{133}$ au sens antique et pré-politique du peuple national dont Carl Schmitt s'est fait le chantre. La souveraineté populaire apparaissant sous les traits de la souveraineté nationale au sein de la réalité historique effectivement advenue de l'État-Nation, Arendt ne peut que rejeter le concept critique de nation sous l'influence fatale de l'interprétation nationaliste de Rousseau par Schmitt. C'est même ce qui la pousse à stigmatiser la Révolution française comme révolution nationale.

\section{Critique de la révolution nationale}

Dans l'essai Sur la révolution, Arendt repère dans le présupposé nationaliste des États-Nations européens l'origine de la destruction totalitaire du politique par le pouvoir de la terreur jacobine, puis du terrorisme idéologique de la révolution russe, exercé sur les individus au nom de la nation unanime. L'État-Nation qui naît historiquement de la Révolution est en effet fondé sur le principe antipolitique de la domination violente, ${ }^{134}$ le monopole de la violence légitime étant exercée au nom de la volonté unanime du peuple qu'il convient d'imposer aux ennemis de la nation par la force du pouvoir révolutionnaire. La Révolution française échoue à instituer la République dans la mesure même où elle engendre l'État-Nation : " ce n'est pas la République, mais la naissance de l'État-Nation, du moins en Europe, qui s'avéra l'unique et irréversible conquête de la Révolution ». ${ }^{135}$ C'est que « les arguments de l'État-Nation ont dès le début dissimulé et refoulé à l'arrière-plan les arguments de la République ». ${ }^{136}$ L'État-Nation, qui se dit Nationalstaat en allemand, se révèle être un État national tendanciellement nationaliste qui s'appuie sur la fiction de l'unanimisme de l'union nationale.

133 lbid., p. 488, trad. p. 511 (il est question d'une communauté nationale).

134 Ibid., Essai sur la révolution (1963), p. 20, trad. p. 22 vs p. 355, trad. p. 408.

135 lbid., p. 66, trad. p. 74.

136 lbid., p. 27 , trad. p. 30. 
Arendt croit pouvoir débusquer ce présupposé nationaliste de la terreur révolutionnaire au cœur même de la conception rousseauiste de la volonté générale : ${ }^{137}$

«En termes politiques, Rousseau présuppose silencieusement l'existence d'un ennemi extérieur par rapport auquel toutes les luttes $d$ 'intérêt à l'intérieur de la nation disparaissent et face auquel la nation devient unanime. Ce n'est qu'à la condition d'un danger immédiat relevant de la politique extérieure qu'il peut y avoir quelque chose comme "la nation une et indivisible", l'idéal du nationalisme français comme de tous les nationalismes. C'est pourquoi l'unité nationale ne peut se réaliser qu'à propos des questions de politique extérieure [...] Mais Rousseau franchit lui-même un pas supplémentaire. L'ennemi extérieur en tant que principe unificateur de la nation ne lui suffisait pas, il voulait que l'unité et l'unanimité s'élèvent de la nation elle-même et deviennent ainsi efficaces en politique intérieure. [...] Le coup de force de cette solution consistait à élever cet ennemi intérieur, caché dans le cœur de chacun, au rang d'un principe unifiant la nation de l'intérieur en additionnant chaque volonté particulière et tous les intérêts en leur somme totale. L'ennemi commun à l'intérieur de la nation est la somme complète des intérêts particuliers de tous ses citoyens ${ }^{138}$

La Révolution est précipitée dans un état de guerre permanente, à l'extérieur comme à l'intérieur, à cause de ce tournant fatal qui l'amène à déplacer "l'accent du type de régime vers "I'incorruptibilité naturelle d'une classe" ou encore de la République vers le peuple ou la nation ». ${ }^{139}$ Robespierre invoque ainsi la volonté générale du peuple dont l'unanimisme est présupposé en lieu et place du consentement de tous qu'il faudrait obtenir par une discussion publique. ${ }^{140} \mathrm{C}^{\prime}$ est le présupposé de la bonté naturelle du peuple ${ }^{141}$ qui lui permet de trancher le nœud gordien de la question sociale en invoquant la pitié comme seule force capable d' " unifier une nation scindée entre riches et pauvres $»^{142}$ et la vertu comme une obligation civique et

137 Ibid., pp. 96-99, trad. pp. 107-111.

138 Ibid., p. 98, trad. p. 110 . p. 393

139 Ibid., p. 116, trad. p. 131.

140 lbid., p. 96, trad. p. 108. pp. 391-392

141 p. 95, p. 75 trad. fr. p. 391.

142 lbid., p. 101, trad. p. 113. 
morale d'être solidaire avec les misérables en identifiant sa propre volonté à la volonté du peuple : il faut une volonté UNE. ${ }^{143}$ Le peuple est considéré comme singulier par rapport à l'infinie diversité des opinions plurielles de la foule. ${ }^{144}$ Loin d'instituer une communauté de citoyens aux opinions divergentes, la révolution a cultivé ce que Sieyès appelle l'intérêt du corps que les personnes privées sont tout naturellement amenées à partager par leur appartenance à un même corps social, le corps social de la Nation au fondement du contrat social :

"La seule chose qui sauve encore et toujours de l'effondrement immédiat les États-nations fondés sur la volonté générale est la fantastique légèreté avec laquelle chaque homme qui désire le fardeau et la gloire de la dictature peut manipuler et se soumettre cette prétendue volonté nationale. La dictature est le type de régime qui est inscrit sur le corps de l'État national, et Napoléon ne fut que le premier, et reste encore et toujours un des plus grands parmi les dictateurs nationaux, qui put déclarer sous les applaudissements de toute la nation: "Je suis le pouvoir constituant". Pourtant, le diktat de la volonté d'un homme en laquelle puisse s'incarner la fiction, inhérente à l'État national, d'une volonté unanime du peuple ne fut jamais nécessaire que dans les périodes de crise; ce n'était pas la volonté, mais l'intérêt, la structure solide de la société de classes qui constitua les véritables fondations de l'État national ». ${ }^{145}$

C'est que la société de classes est tenue solidement par une solidarité, nationale, qui contredit et refoule la lutte des classes : national-social en quelque sorte, l'intérêt social est indissociablement national dans la mesure même où, démocratique, l'État national s'attache à satisfaire le bien-être du peuple ou des masses, c'est-à-dire leur intérêt. Si l'État national s'avère être fatal pour la république, c'est qu'il place l'intérêt au cœur du contrat social pour résoudre de manière volontariste les contradictions sociales au sein du corps de la nation. Mais le principe absolutiste ${ }^{146}$ de la souveraineté

$143 \mathrm{lbid} .$, p. 94, trad. p. 106. La citation est en français dans le texte allemand comme américain.

144 Ibid., p. 118 , trad. p. 133.

145 Ibid., p. 212, trad. p. 240.

146 Ibid., p. 27, trad. p. 30. 
nationale ${ }^{147}$ ne peut engendrer la fiction unanimiste qu'à condition d'être représenté par un souverain en chair et en os : le contrat social implique tout à la fois le principe absolutiste d'un renoncement au pouvoir au profit de l'État (Hobbes) et « le principe de l'État national qui a besoin du souverain en tant que représentant de la nation toute entière, pour incarner la prétendue volonté générale ». ${ }^{148}$

Loin d'être spontanée, l'unanimité (Einmütigkeit) des esprits (Gemüt), qui $s^{\prime}$ exprime dans le sentiment national ${ }^{149}$ et se déchaîne même en passion nationale, est fabriquée du jour au lendemain par l'homme fort du régime qui prétend parler au nom de la nation : "cette "opinion publique" unanime pas moins hystérique est la mort de toutes les opinions ». ${ }^{150}$ Car la « domination de l'opinion publique » que les fondateurs de la république américaine jugent tyrannique présuppose de considérer le peuple comme un singulier collectif, et non comme un pluriel comprenant l'infinie diversité d'une multitude d'opinions : «la prétendue opinion publique leur était suspecte justement parce que, par hystérie ou manipulation, elle mène facilement à l'unanimité ». ${ }^{151}$ Carl Schmitt est ici visé qui soutenait que l'acclamation est la « forme naturelle de l'expression immédiate de la volonté d'un peuple $»^{152}$ par laquelle la foule, assemblée sur la place publique, ${ }^{153}$ se manifeste " en tant qu' "opinion publique" » en approuvant ou en rejetant. ${ }^{154}$ En répondant à Schmitt que " la seule véritable forme de démocratie immédiate $»^{155}$ est celle du peuple discutant et argumentant ${ }^{156}$ dans les conseils de communes, ${ }^{157}$ Arendt prend à revers la critique schmittienne en reprochant au système parlementaire de tendre à devenir un système plébiscitaire qui ne concède comme droit à l'électeur « que d'approuver

147 Ibid., p. 251, trad. p. 288.

148 Ibid., p. 222, trad. p. 252.

149 Ibid., p. 125, trad. p. 141.

150 lbid., p. 294, trad. p. 337.

151 lbid., pp. 118-119, trad. p. 133.

152 Voir SCHMITT, Verfassungslehre, p. 83, trad. p. 219.

153 Ibid., p. 243, trad. p. 382.

154 lbid., p. 84, trad. p. 219.

155 ARENDT, Sur la révolution, p. 331, trad. p. 381.

156 lbid., p. 110 , trad. p. 123.

157 Ibid., p. 302, trad. p. 348. 
ou rejeter le choix déjà fait » par le parti des candidats à l'élection, ${ }^{158}$ là où Schmitt approuve la transformation du système parlementaire en démo-

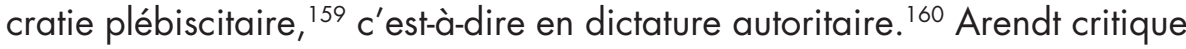
ainsi la dictature de l'opinion publique unanime qui permet à l'oligarchie parlementaire ou révolutionnaire, au nom du peuple qui la plébiscite, $d^{\prime}{ }^{\prime} i m-$ poser la volonté nationale par un pouvoir dictatorial :

«[...] la dictature du parti unique constitue non seulement le stade provisoirement ultime de l'État-Nation, mais encore la conséquence tout aussi logique du système multipartite $» .{ }^{161}$

La dictature apparaît comme l'aboutissement logique de la révolution nationale dont la Révolution française constituerait ainsi le prototype. ${ }^{162}$ En disqualifiant la Révolution comme nationale, Arendt signale l'empreinte que le contre-modèle de la révolution nationale de l'Allemagne nazie exerce en retour sur son interprétation de la révolution française comme de toutes les révolutions socialistes qui l'ont prise pour modèle. ${ }^{163} \mathrm{Ce}$ qu'Arendt reproche

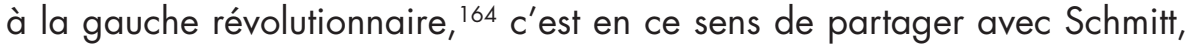
qui s'inspirait moins de Marx et Lénine que de Clausewitz, l'idéologie de la dictature révolutionnaire: ${ }^{165}$ l'apologie antipolitique d'une violence qui devient, à travers la Révolution ou la guerre, un moyen de poursuivre la politique. S'étant formés dans le cadre des États-nations, les partis de gauche révolutionnaire ont ainsi partagé avec les puissances dominantes une même conception de l'identité d'essence entre puissance et pouvoir ${ }^{166}$ (Macht und Gewalt) :

158 Ibid., p. 356, trad. p. 410. Arendt emploie les mêmes verbes que Schmitt : zustimmen et ablehnen.

159 SCHMITT, Legalität und Legitimität, pp. 85-87, 13-14.

160 lbid., p. 68-81, 87.

161 ARENDT, Sur la révolution, p. 342, trad. p. 393.

$162 \mathrm{lbid} .$, p. 206, trad. p. 232.

163 Ibid., p. 206, 312, 317, trad. pp. 232, 360, 366.

164 Ibid., p. 352, trad. p. 405, cf. p. 318 , trad. p. 366.

$165 \mathrm{lbid} .$, p. 318 , trad. p. 366.

166 Ibid., p. 328 , trad. p. 379. 
"Comme Marx et Lénine ont grandi dans la tradition de l'État-Nation $[\ldots]$, ils ne se représentaient rien d'autre sous une révolution qu'une prise de pouvoir et ils identifiaient la puissance avec le monopole des moyens étatiques de pouvoir ».

Dans ces conditions, la gauche révolutionnaire $n^{\prime}$ a pu finalement que sombrer dans le totalitarisme de la dictature du parti unique ${ }^{167}$ des démocraties populaires. ${ }^{168}$ Le multipartisme du système parlementaire d'Europe continentale ayant pour conséquence logique d'engendrer la dictature d'un parti unique, cela revient à reconnaître que la démocratie populaire apparaît à I'horizon de l'État national-social des démocraties bourgeoises parlementaires comme leur point d'aboutissement. L'anti-modèle national-socialiste comme prototype de l'État totalitaire s'avère donc être au fondement de la critique arendtienne de la dictature (national-communiste) des démocraties populaires comme de l'État national-social des démocraties libérales.

Hannah Arendt rejette en fin de compte le concept critique de nation, dont le caractère émancipatoire est récusé, pour une double raison : le potentiel nationaliste que l'État-Nation a historiquement développé et la potentialité dictatoriale qu'implique l'union nationale des masses révolutionnaires derrière son chef. L'unanimisme de la nation révolutionnaire disparaît de la sorte au profit de la pluralité d'un peuple républicain en mouvement politique de dissension. ${ }^{169}$ L'option de la nation républicaine ayant été écartée au profit d'un peuple chargé d'assumer la puissance émancipatrice, le concept de nation ne peut plus être que dans un état critique.

\section{BibLIOGRAPHIE}

ARENDT, Hannah (1933), Rahel Varnhagen, Munich, Piper, 2003.

Trad. fr., Rahel Varnhagen (2016), Payot, "Petite Bibliothèque », Paris. ARENDT, Hannah (1946), Die verborgene Tradition, Suhrkamp, 1976. ARENDT, Hannah (2000), Vor Antisemitismus ist man nur noch auf dem Monde sicher, Munich, Piper (trad. fr., Auschwitz et Jerusalem, Presses Pocket, Paris, 1993).

167 Ibid., n. 43 p. 384 , trad. n. 40 p. 441.

$168 \mathrm{lbid} .$, p. 340 , trad. p. 391.

169 Voir Christian FERRIÉ (2007), « Le pouvoir du peuple entre nation et fédération », in TASSIN, Hannah Arendt. Crises de l'État-Nation, Sens\&Tonka, pp. 69-72. 
ARENDT, Hannah (1955), Elemente und Ursprünge totaler Herrschaft, Piper, Munich, 1986 (trad. fr., Les Origines du totalitarisme. Eichmann à Jérusalem, Paris, Gallimard, "Quarto », 2002).

ARENDT, Hannah (1963), Über die Revolution, Piper, Munich, 1974 (trad. fr. Essai sur la révolution, Gallimard, Paris, 1967).

ARENDT, Hannah (1994), Essays in Understanding, Schocken Books.

RENAN, Ernest, Qu'est-ce qu'une nation? (11 mars 1882), Pocket, Paris, 1992.

SCHMITT, Carl (1923), Die geistesgeschichtliche Lage des heutigen Parlementarismus, Duncker \& Humblot, Berlin, 8e édition 1996 (trad. fr. Parlementarisme et démocratie, Sevil, 1988).

SCHMITT, Carl, Verfassungslehre (1928), Duncker \& Humblot, Berlin, 8 e édition 1993 (trad. fr. Théorie de la constitution, PUF, 1993).

SCHMITT, Carl, Legalität und Legitimität (1932), Duncker \& Humblot, Berlin, 6e édition 1998.

SCHMITT, Carl, Der Begriff des Politischen (1932), Duncker \& Humblot, Berlin, 1963 (trad. fr. La notion de politique, Calmann-Lévy, 1972).

SCHMITT, Carl, Staat, Bewegung, Volk (1933), Hanseatische Verlagsanstalt Hamburg (trad. fr. État, mouvement, peuple, Paris, KIMÉ, 1997).

SCHMITT, Carl, Positionen und Begriffe im Kampf mit Weimar - Genf - Versailles 1923-1939 (1940), Hanseatische Verlagsanstalt Hamburg (trad. fr. Parlementarisme et démocratie, Paris, Sevil, 1988).

SCHMITT, Carl, Theorie des Partisanen (1963), Duncker \& Humblot, Berlin, 1995 (trad. fr. Théorie du partisan, Paris, Calmann-Lévy, 1972). 